\title{
Partial and total dielectronic recombination rate coefficients for $\mathrm{W}^{73+}$ to $\mathrm{W}^{56+}$
}

\author{
S. P. Preval, ${ }^{*}$ N. R. Badnell, and M. G. O’Mullane \\ Department of Physics, University of Strathclyde, Glasgow G4 ONG, United Kingdom
}

(Received 20 December 2015; published 7 April 2016)

\begin{abstract}
Dielectronic recombination (DR) is a key atomic process that affects the spectroscopic diagnostic modeling of tungsten, most of whose ionization stages will be found somewhere in the ITER fusion reactor: in the edge, divertor, or core plasma. Accurate DR data are sparse while complete DR coverage is unsophisticated (e.g., average-atom or Burgess General Formula), as illustrated by the large uncertainties that currently exist in the tungsten ionization balance. To this end, we present a series of partial final-state-resolved and total DR rate coefficients for $\mathrm{W}^{73+}$ to $\mathrm{W}^{56+}$ tungsten ions. This is part of a wider effort within The Tungsten Project to calculate accurate dielectronic recombination rate coefficients for the tungsten isonuclear sequence for use in collisional-radiative modeling of finite-density tokamak plasmas. The recombination rate coefficients have been calculated with AUTOSTRUCTURE using $\kappa$-averaged relativistic wave unctions in level resolution (intermediate coupling) and configuration resolution (configuration average). Comparison with previous calculations of total DR rate coefficients for $\mathrm{W}^{63+}$ and $\mathrm{W}^{56+}$ yield agreement to within $20 \%$ and $10 \%$, respectively, at peak temperature. It is also seen that the Jüttner correction to the Maxwell distribution has a significant effect on the ionization balance of tungsten at the highest charge states, changing both the peak abundance temperatures and the ionization fractions of several ions.
\end{abstract}

DOI: 10.1103/PhysRevA.93.042703

\section{INTRODUCTION}

ITER [1] is posited to be the penultimate step in realizing a nuclear fusion power plant. It will be significantly larger than present machines, such as JET, in terms of plasma volume, core temperature, and physical size [2]. Beryllium-coated tiles will line the wall of the main reactor vessel due to their low erosion rate and the low tritium retention of Be. Tungsten $(Z=74)$ will be used in regions of high power-loads, such as the divertor chamber at the base of the main vessel, and it is also resistant to tritiation [3]. On the downside, such high- $Z$ elements are efficient radiators and must be kept to a minimum in the main plasma to avoid degrading its confinement. Because of this, JET has undergone a major upgrade to an ITER-like wall to act as a test-bed. Control of tungsten sources and its subsequent transport are under intensive study [4]. Tungsten is the highest- $Z$ metal in widespread use in a tokamak. Prior to the installation of the ITER-like wall at JET, molybdenum $(Z=42)$ was the highest- $Z$ metal in widespread use, at Alcator C-Mod. Like tungsten, molybdenum has a low tritium absorption rate [5]. However, molybdenum has a significantly lower melting point than tungsten, and it also transmutes to technetium, complicating reactor decommissioning.

Most of the tungsten isonuclear sequence needs to be covered by non-LTE plasma modeling, from its initial sputtering from surfaces through the edge, divertor, and core plasmas. One of the most basic quantities is the tungsten ionization balance: a measure of the dominant ionization stages as a function of temperature and density. While our

\footnotetext{
*simon.preval@strath.ac.uk

Published by the American Physical Society under the terms of the Creative Commons Attribution 3.0 License. Further distribution of this work must maintain attribution to the author(s) and the published article's title, journal citation, and DOI.
}

understanding of the required ionization rates appears to be in reasonable order [6], the same is not true for the competing dielectronic plus radiative recombination rates $(D R+R R)$. In Fig. 1, we compare the zero-density ionization balance for tungsten obtained using two different sets of recombination data $[7,8]$ and the same ionization rate coefficients of [6]. It can be seen that there are large discrepancies between the peak temperatures of individual ionization stages as well as the fractional population of said ionization stage. The dielectronic recombination data of Pütterich et al. [7] were calculated with ADPAK $[9,10]$ using an average-atom model, and they were scaled by the authors in order to improve agreement between theory and experiment with regard to the shape of the fractional abundances of $\mathrm{W}^{22+}-\mathrm{W}^{55+}$. The DR data of Foster [8] used the Burgess General Formula [11]. Both used the same scaled hydrogenic radiative recombination data. Clearly, more reliable DR data are required.

Another issue is that the magnetic fusion plasmas cannot be taken to be a zero-density plasma. The true ionization balance is density-dependent, and the corresponding densitydependent (effective) ionization and recombination rate coefficients are obtained from collisional-radiative modeling. The ionization rate coefficients are much less sensitive to density effects than the recombination ones because dielectronic recombination takes place through and to high-Rydberg states. Therefore, partial final-state-resolved rate coefficients are needed. Even where detailed calculations have been made, the data available are usually in the form of zero-density totals, i.e., summed over all final states. As such, it is difficult to use such data for collisional-radiative modeling in a reliable manner.

Detailed calculations have been performed for a select few ions of tungsten. However, these are very sparse and tend to be for closed-shell ions, which are important for plasma diagnostics. The most complicated exception to date is our work on the open $f$-shell: $\mathrm{W}^{20+, 18+}\left(4 d^{10} 4 f^{q}, q=\right.$ $8,10)[12,13]$. Data for these ions were calculated using an 


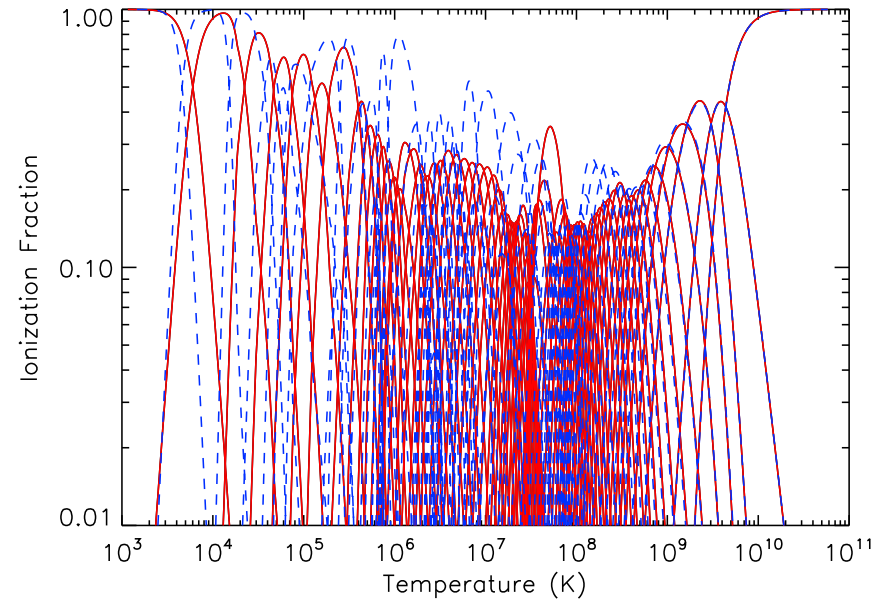

FIG. 1. Zero-density fractional abundances of tungsten ionization stages, calculated using the combination data of Pütterich et al. [7] (red, solid curves) and Foster [8] (blue, dashed curves). Both use the ionization rate coefficients from Loch et al. [6].

upgraded version of AUTOSTRUCTURE designed to handle the increased complexity of the problem. The HULLAC [14] and the COWAN code [15] have been used by Safronova et al. [16-20] to calculate DR rate coefficients for $\mathrm{W}^{5+}, \mathrm{W}^{28+}, \mathrm{W}^{46+}, \mathrm{W}^{63+}$, and $\mathrm{W}^{64+}$, respectively. Behar et al. [21] and Peleg et al. [22] have also used these codes for $\mathrm{W}^{46+}, \mathrm{W}^{64+}$, and $\mathrm{W}^{56+}$, respectively. In addition, the Flexible Atomic Code (FAC [23]) has been used by Meng et al. [24] and Li et al. [25] to calculate DR rate coefficients for $\mathrm{W}^{47+}$ and $\mathrm{W}^{29+}$, respectively. Just recently, Wu et al. [26] calculated zero-density total DR rate coefficients for $\mathrm{W}^{37+}-\mathrm{W}^{46+}$ using FAC.

In contrast, partial RR rate coefficients have been calculated for the entire isonuclear sequence of tungsten, and the results were presented in a series of papers by Trzhaskovskaya et al. [27-30]. The authors used a Dirac-Fock method with fully relativistic wave functions, and they included contributions from all significant radiation multipoles. They stated that the majority of their RR rate coefficients were calculated to $<1 \%$ numerical accuracy. However, for outer shell RR and high temperatures, their rate coefficients were calculated to $<5 \%$ [27]. The authors also presented total RR rate coefficients summed up to $n=20$ and $\ell=19$.

To address this situation, we have embarked on a programme of work, as part of The Tungsten Project, which aims to calculate partial final-state resolved DR rate coefficients for use in collisional-radiative modeling with atomic data and analysis structure (ADAS) [31] for the entire isonuclear sequence of tungsten. For completeness and ease of integration within ADAS, we compute the corresponding RR data at the same time. Zero-density totals are readily obtained from the archived data. The work presented here covers $\mathrm{W}^{73+}$ to $\mathrm{W}^{56+}$.

On a practical technical point, the names of various elements in the Periodic Table are not particularly helpful to label ionization stages of a large isonuclear sequence such as tungsten. Thus, we will not refer to such species by a name such as Pr-like. Instead, we adopt a notation based on the number of electrons possessed by a particular ion. For example, H-like (1 electron) $\mathrm{W}^{73+}$ will be referred to as 01-like, Ne-like (10 electrons) $\mathrm{W}^{64+}$ will be referred to as 10-like, and Pr-like (59 electrons) $\mathrm{W}^{15+}$ as 59-like. This mirrors our database archiving.

The outline of this paper is as follows: In Sec. II, we outline the background theory for our description of DR and RR, as encapsulated in the AUTOSTRUCTURE code, and we give consideration to the delivery of data in a manner appropriate for collisional-radiative modeling. In Sec. III, we describe our calculations for 00-like to 18-like ions. In Sec. IV, we present our results for DR or RR rate coefficients and compare them with those published previously, where available; then we look at how the zero-density ionization balance of tungsten changes upon using our new recombination data. We conclude with some final remarks, and we outline future calculations.

\section{THEORY}

We use the distorted-wave atomic package AUTOSTRUCTURE [32-34]. For recombination, AUTOSTRUCTURE makes use of the independent processes and isolated resonance approximations [35]. Then, the partial DR rate coefficient ${ }^{\mathrm{DR}} \alpha_{f v}^{z+1}$, from some initial state $v$ of ion $X^{+z+1}$ to a final state $f$ of ion $X^{+z}$, can be written as

$$
\begin{aligned}
\mathrm{DR}_{\alpha_{f \nu}}^{z+1}\left(T_{e}\right)= & \left(\frac{4 \pi a_{0}^{2} I_{H}}{k_{B} T_{e}}\right)^{\frac{3}{2}} \sum_{j} \frac{\omega_{j}}{2 \omega_{\nu}} \exp \left[-\frac{E}{k_{B} T_{e}}\right] \\
& \times \frac{\sum_{l} A_{j \rightarrow \nu, E l}^{a} A_{j \rightarrow f}^{r}}{\sum_{h} A_{j \rightarrow h}^{r}+\sum_{m, l} A_{j \rightarrow m, E l}^{a}},
\end{aligned}
$$

where the $A^{a}$ are the autoionization rates, $A^{r}$ are the radiative rates, $\omega_{j}$ is the statistical weight of the $N$-electron target ion, and $E$ is the total energy of the continuum electron, minus its rest energy, and with corresponding orbital angular momentum quantum number $l$ labeling said channels. $I_{H}$ is the ionization energy of the hydrogen atom, $k_{B}$ is the Boltzmann constant, $T_{e}$ is the electron temperature, and $\left(4 \pi a_{0}^{2}\right)^{3 / 2}=$ $6.6011 \times 10^{-24} \mathrm{~cm}^{3}$. The sum over the autoionizing states $j$ recognizes the fact that, in general, these states have sufficiently short lifetimes in a magnetic fusion plasma for them not to be collisionally redistributed before breaking up, although statistical redistribution is assumed in some cases [36].

The partial RR rate coefficient ${ }^{\mathrm{RR}} \alpha_{f v}^{z+1}$ can be written, in terms of the photoionization cross section ${ }^{\mathrm{PI}} \sigma_{v f}^{z}$ for the inverse process using detailed balance, as

$$
\begin{aligned}
\mathrm{RR}_{\alpha_{f \nu}^{z+1}}^{z+1}\left(T_{e}\right)= & \frac{c \alpha^{3}}{\sqrt{\pi}} \frac{\omega_{f}}{2 \omega_{v}}\left(I_{H} k_{B} T_{e}\right)^{-3 / 2} \\
& \times \int_{0}^{\infty} E_{v f}^{2}{ }^{\mathrm{PI}} \sigma_{v f}^{z}(E) \exp \left[-\frac{E}{k_{B} T_{e}}\right] d E,
\end{aligned}
$$

where $E_{v f}$ is the corresponding photon energy, and $c \alpha^{3} / \sqrt{\pi}=6572.67 \mathrm{~cm} \mathrm{~s}^{-1}$ for ${ }^{\mathrm{PI}} \sigma_{v f}^{z}$ given in $\mathrm{cm}^{2}$. The photoionization cross sections for arbitrary electric and magnetic multipoles are given by [37]. The numerical approaches to converging the quadrature accurately and efficiently have been given previously [36].

At high temperatures $\left(\gtrsim 10^{9} \mathrm{~K}\right)$, relativistic corrections to the usual Maxwell-Boltzmann distribution become important. The resultant Maxwell-Jüttner distribution [38] reduces simply 
to an extra multiplicative factor, $F_{\mathrm{r}}(\theta)$, to be applied to the Maxwell-Boltzmann partial rate coefficients:

$$
F_{\mathrm{r}}(\theta)=\sqrt{\frac{\pi \theta}{2}} \frac{1}{K_{2}(1 / \theta) e^{1 / \theta}},
$$

where $\theta=\alpha^{2} k_{B} T / 2 I_{H}, \alpha$ is the fine-structure constant, and $K_{2}$ is the modified Bessel function of the second kind. This factor is normally consistently omitted from data archived in ADAS, being subsequently applied if required in extreme cases. However, since it has a non-negligible affect at the temperature of peak abundance for the highest charge states, we consistently include it for all tungsten DR and RR data and flag this in the archived files.

Plasma densities in magnetic fusion reactors vary greatly. For ITER, the plasma densities are thought to vary from $10^{10}$ to $10^{13} \mathrm{~cm}^{-3}$ for the edge plasma, to $\sim 10^{14} \mathrm{~cm}^{-3}$ for the core plasma, reaching $\sim 10^{15} \mathrm{~cm}^{-3}$ for the divertor plasma. Because of these densities, the coronal picture breaks down: capture into an excited state does not cascade down to the ground uninterrupted. Instead, further collisions take place, leading in particular to stepwise ionization, for example. This strongly suppresses coronal total recombination rate coefficients. Collisional-radiative (CR) modeling of the excited-state population distribution is necessary. This leads to density-dependent effective ionization and recombination-rate coefficients. A key ingredient for CR modeling is partial final-state-resolved recombination data. Our approach for light systems is detailed in [39] and [36] for DR and RR, respectively. Low-lying final states are fully level-resolved while higher-lying states are progressively ( $n \ell-$ and $n-$ ) bundled over their total quantum numbers while retaining their level parentage. Initial ground and metastable levels are also fully resolved. The data are archived in ADAS standard formats, viz., adf09 (DR) and adf48 (RR). One does not need to progress far into the $M$-shell for the number of such final states to become unmanageable by $\mathrm{CR}$ modeling and for further bundling to become necessary. This is carried out most efficiently as the partial recombination rate coefficients are calculated, and it leads to much more compact adf09 and adf48 files. We find it necessary to bundle over all final recombined levels within a configuration. For such configurations that straddle the ionization limit, we include the statistical fractions within the adf files. The initial ground and metastable levels remain level-resolved, as does the calculation of autoionizing branching ratios (fluorescence yields). We describe such a mixed resolution scheme as a "hybrid" approach, and the adf files are labeled accordingly. All resultant adf09 and adf48 files are made available via OPEN-ADAS [40].

\section{CAlCulations}

All rates and cross sections were determined upon solving the $\kappa$-averaged quasi-one-electron Dirac equation for the large and small components utilizing the Thomas-Fermi-DiracAmaldi model potential [41] with unit scaling parameters to represent the $N$-and $(N+1)$-electron ions. We utilized several coupling schemes. Configuration average (CA) was used to give a quick overview of the problem. This neglects configuration mixing and relativistic interactions in the Hamiltonian.
$L S$ coupling (LS) allows for configuration mixing but tends to overestimate it in such highly charged ions because relativistic interactions push interacting terms farther apart. Thus, our main body of data are calculated in intermediate coupling (IC). For the $K$-shell ions, we included valence-valence two-body fine-structure interactions. These gave rise to an $\sim 7 \%$ increase in the total DR rate coefficients for 01-like and 02-like ions at high temperatures. We neglect these interactions for the $L$ - and $M$-shell ions since the increase in the total DR rate coefficient is $<1 \%$.

\section{A. DR}

It is necessary to include all dominant DR reactions illustrated by Eq. (1). The initial state $v$ is taken to be the ground state. Metastables are unlikely to be important at such high charge states. The driving reactions are the autoionizing states produced by one-electron promotions from the ground configuration, with a corresponding capture of the continuum electron. We label these core excitations by the initial $(n)$ and final $\left(n^{\prime}\right)$ principal quantum numbers of the promoted electron, and we include all corresponding subshells ( $\ell$ values). The dominant contributions come from $\Delta n=0\left(n=n^{\prime}\right)$ and $\Delta n=1\left(n^{\prime}=n+1\right)$, being well separated in energy and temperature. Contributions from $\Delta n>1$ tend to be suppressed by autoionization into excited states, as represented by the sum over $A^{a}$ in the denominator of (1). The outermost shell dominates but the $\Delta n=1$ inner-shell promotion $\left(n=n^{\prime}-1\right)$ can be significant when there are few outer $n$-shell electrons. As their number increases, core rearranging autoionizing transitions suppress this inner-shell contribution. These core excitations define a set of $N$-electron configurations to which continuum and Rydberg electrons are coupled.

Based on these promotion rules, the core excitations considered for each ion $\left(\mathrm{W}^{73+}\right.$ to $\left.\mathrm{W}^{56+}\right)$ are listed in Table I. The calculations were carried out first in CA to determine which excitations are dominant. We omitted core excitations that contribute $<1 \%$ to the sum total of all DR core-excitation rate coefficients spanning the ADAS temperature grid. This grid covers $10 z^{2}-2 \times 10^{6} z^{2} \mathrm{~K}$, where $z$ is the residual charge of the initial target ion. DR for the dominant core excitations is then calculated in IC. The $n \ell$ Rydberg electron, in the sum over autoionizing states $j$, is calculated explicitly for each principal quantum number up to $n=25$ and then on a quasilogarithmic $n$

TABLE I. Core excitations included in the DR rate coefficient calculations for $\mathrm{W}^{73+}$ to $\mathrm{W}^{56+}$. All core excitations have been calculated in IC and CA.

\begin{tabular}{|c|c|c|c|c|c|}
\hline Ionlike & Symbol & Core excitations & Ion & Symbol & Core excitations \\
\hline 01-like & $\mathrm{W}^{73+}$ & $1-2,1-3$ & 10-like & $\mathrm{W}^{64+}$ & $2-3,2-4$ \\
\hline 02-like & $\mathrm{W}^{72+}$ & $1-2,1-3$ & 11-like & $\mathrm{W}^{63+}$ & $2-3,3-3,3-4,3-5$ \\
\hline 03-like & $\mathrm{W}^{71+}$ & $1-2,2-2,2-3,2-4$ & 12-like & $\mathrm{W}^{62+}$ & $2-3,3-3,3-4,3-5$ \\
\hline 04-like & $\mathrm{W}^{70+}$ & $1-2,2-2,2-3,2-4$ & 13-like & $\mathrm{W}^{61+}$ & $2-3,3-3,3-4,3-5$ \\
\hline 05-like & $\mathrm{W}^{69+}$ & $2-2,2-3$ & 14-like & $\mathrm{W}^{60+}$ & $2-3,3-3,3-4,3-5$ \\
\hline 06-like & $\mathrm{W}^{68+}$ & $2-2,2-3$ & 15-like & $\mathrm{W}^{59+}$ & $2-3,3-3,3-4,3-5$ \\
\hline 07-like & $W^{67+}$ & $2-2,2-3$ & 16-like & $\mathrm{W}^{58+}$ & $2-3,3-3,3-4,3-5$ \\
\hline 08-like & $\mathrm{W}^{66+}$ & $2-2,2-3$ & 17-like & $\mathrm{W}^{57+}$ & $2-3,3-3,3-4,3-5$ \\
\hline 09-like & $\mathrm{W}^{65+}$ & $2-2,2-3$ & 18-like & $\mathrm{W}^{56+}$ & $2-3,3-3,3-4,3-5$ \\
\hline
\end{tabular}


mesh up to $n=999$. The partial DR rate coefficient tabulation is based on this mesh of $n$ values. Total (zero-density) DR rate coefficients are obtained by interpolation and quadrature of these partials. The maximum Rydberg orbital angular momentum $(\ell)$ is taken to be such that the total rate coefficients are converged to better than $1 \%$ over the ADAS temperature range. Radiative transitions of the Rydberg electron to final states with principal quantum number greater than that of the core excitations are described hydrogenically. Those in the core are described by a set of $(N+1)$-electron configurations that are generated by adding another core-electron orbital to all $N$-electron configurations describing the core excitations. In the case of $\Delta n>1$ core excitations, this also allows for dielectronic capture into the core.

To make clear the complete set of configurations included for a typical calculation, we give a list of configurations used to calculate DR rate coefficients for 14-like 3-3 and 3-4 core excitations in Table II. We have marked with an asterisk configurations that were added to allow for the dominant configuration mixing within a complex by way of the "one up, one down rule." For example, the configuration $3 s 3 p^{2} 3 d$ strongly mixes with $3 p^{4}$ and $3 s^{2} 3 d^{2}$.

TABLE II. Set of configurations used for the 14-like 3-3 and 3-4 core-excitation calculations. Configurations marked with an asterisk were included as mixing configurations.

\begin{tabular}{|c|c|c|c|c|}
\hline $\begin{array}{l}3-3 \\
N \text {-electron }\end{array}$ & $\begin{array}{l}(N+1)- \\
\text { electron }\end{array}$ & $\begin{array}{c}3-4 \\
N \text {-electron }\end{array}$ & $\begin{array}{l}(N+1)- \\
\text { electron }\end{array}$ & \\
\hline $\begin{array}{l}3 s^{2} 3 p^{2} \\
3 s^{2} 3 p 3 d \\
3 s 3 p^{3} \\
3 s 3 p^{2} 3 d \\
* 3 p^{4} \\
* 3 s^{2} 3 d^{2}\end{array}$ & $\begin{array}{l}3 s^{2} 3 p^{3} \\
3 s^{2} 3 p^{2} 3 d \\
3 s^{2} 3 p 3 d 2 \\
3 s 3 p^{4} \\
3 s 3 p^{3} 3 d \\
3 s 3 p^{2} 3 d^{2} \\
* 3 p^{5} \\
* 3 p^{4} 3 d \\
* 3 s^{2} 3 d^{3}\end{array}$ & $\begin{array}{l}3 s^{2} 3 p^{2} \\
3 s^{2} 3 p 3 d \\
3 s^{2} 3 p 4 s \\
3 s^{2} 3 p 4 p \\
3 s^{2} 3 p 4 d \\
3 s^{2} 3 p 4 f \\
3 s 3 p^{3} \\
3 s 3 p^{2} 3 d \\
3 s 3 p^{2} 4 s \\
3 s 3 p^{2} 4 p \\
3 s 3 p^{2} 4 d \\
3 s 3 p^{2} 4 f \\
* 3 p^{4} \\
* 3 s^{2} 3 d^{2} \\
* 3 s^{2} 3 d 4 s \\
* 3 s^{2} 3 d 4 p \\
* 3 s^{2} 3 d 4 d \\
* 3 s^{2} 3 d 4 f\end{array}$ & $\begin{array}{l}3 s^{2} 3 p^{2} 4 s \\
3 s^{2} 3 p^{2} 4 p \\
3 s^{2} 3 p^{2} 4 d \\
3 s^{2} 3 p^{2} 4 f \\
3 s^{2} 3 p 3 d 4 s \\
3 s^{2} 3 p 3 d 4 p \\
3 s^{2} 3 p 3 d 4 d \\
3 s^{2} 3 p 3 d 4 f \\
3 s 3 p^{3} 4 s \\
3 s 3 p^{3} 4 p \\
3 s 3 p^{3} 4 d \\
3 s 3 p^{3} 4 f \\
3 s 3 p^{2} 3 d 4 s \\
3 s 3 p^{2} 3 d 4 p \\
3 s 3 p^{2} 3 d 4 d \\
3 s 3 p^{2} 3 d 4 f \\
* 3 p^{4} 4 s \\
* 3 p^{4} 4 p \\
* 3 p^{4} 4 d \\
* 3 p^{4} 4 f \\
* 3 s^{2} 3 d^{2} 4 s \\
* 3 s^{2} 3 d^{2} 4 p \\
* 3 s^{2} 3 d^{2} 4 d \\
* 3 s^{2} 3 d^{2} 4 f\end{array}$ & $\begin{array}{l}3 s^{2} 3 p 4 s^{2} \\
3 s^{2} 3 p 4 s 4 p \\
3 s^{2} 3 p 4 s 4 d \\
3 s^{2} 3 p 4 s 4 f \\
3 s^{2} 3 p 4 p^{2} \\
3 s^{2} 3 p 4 p 4 d \\
3 s^{2} 3 p 4 p 4 f \\
3 s^{2} 3 p 4 d^{2} \\
3 s^{2} 3 p 4 d 4 f \\
3 s^{2} 3 p 4 f^{2} \\
3 s 3 p^{2} 4 s^{2} \\
3 s 3 p^{2} 4 s 4 p \\
3 s 3 p^{2} 4 s 4 d \\
3 s 3 p^{2} 4 s 4 f \\
3 s 3 p^{2} 4 p^{2} \\
3 s 3 p^{2} 4 p 4 d \\
3 s 3 p^{2} 4 p 4 f \\
3 s 3 p^{2} 4 d^{2} \\
3 s 3 p^{2} 4 d 4 f \\
3 s 3 p^{2} 4 f^{2} \\
* 3 s^{2} 3 d 4 s^{2} \\
* 3 s^{2} 3 d 4 s 4 p \\
* 3 s^{2} 3 d 4 s 4 d \\
* 3 s^{2} 3 d 4 s 4 f \\
* 3 s^{2} 3 d 4 p^{2} \\
* 3 s^{2} 3 d 4 p 4 d \\
* 3 s^{2} 3 d 4 p 4 f \\
* 3 s^{2} 3 d 4 d^{2} \\
* 3 s^{2} 3 d 4 d 4 f \\
* 3 s^{2} 3 d 4 f^{2}\end{array}$ \\
\hline
\end{tabular}

\section{B. $\mathbf{R R}$}

The partial RR rate coefficients were calculated in a similar, but simplified, fashion to $\Delta n=0 \mathrm{DR}$, viz., the $N$-electron target configurations were restricted to those that mixed with the ground and the $(N+1)$-electron configurations were these $N$-electron configurations with an additional core electron. The Rydberg $n \ell$ values were again calculated for each $n$ up to $n=25$ and then on the same $n$-mesh as used for DR, up to $n=$ 999 , with $\ell=0-10$, relativistically. At high $-T\left(>10^{9}\right) \mathrm{K}$, many multipoles contribute to the photoionization or recombination at correspondingly high energies [42]. We included up to E40 in CA and E40/M39 in the IC calculations, which is sufficient to converge the total RR rate coefficients to $<1 \%$ over the ADAS temperature range. A nonrelativistic (dipole) top-up was then used to include up to $\ell=150$ to converge the low-temperature RR rate coefficients-relativistic effects being negligible there. This approach is sufficient to calculate the total RR rate coefficients to better than $1 \%$ numerically.

\section{RESULTS AND DISCUSSION}

In this section, we present the results of our DR and $\mathrm{RR}$ rate coefficient calculations for 00-like to 18-like. In our plots, we show the tungsten fractional peak abundance curves from Pütterich et al. [7] to give an indication of the relevant temperatures for application purposes. At these temperatures, RR is dominated by capture into the lowest available $n l$-subshell. We consider the DR rate coefficients first, and we look at the $K-, L-$, and $M$-shells in turn. Next, we consider the RR rate coefficients, and we assess their importance relative to DR. We compare our results with others where possible. Finally, we look at the effect on the zero-density ionization balance of tungsten when using our new data.

\section{A. $K$-shell DR}

The DR rate coefficients for 01- and 02-like are very small compared to RR. The reason for this is that the RR rate coefficient scales as $z$ (residual charge) while the DR rate coefficient here scales as $z^{-3}$, being proportional to the dielectronic capture rate [the fluorescence yields are close to unity due to the $z^{4}$ scaling of the radiative rates and the $z^{0}(=1)$ of the autoionization]. In Fig. 2, we have plotted the DR and RR rate coefficients for 01-like. In the top subplot, we show the individual contributions from each DR core excitation, and RR. The ionization balance for 01-like, calculated using the scaled recombination data of Pütterich et al. [7] and the ionization data of Loch et al. [6], is plotted also for reference. In the bottom subplot, we have plotted the cumulative sum of each contribution to the total recombination-rate coefficient. This was calculated by taking the fraction of the largest contribution to the total recombination-rate coefficient. The next curve is calculated by adding the first and second largest contributions together, and taking the fraction of this to the total recombination-rate coefficient, and so on. It can be seen that the total recombination-rate coefficient is dominated by $\mathrm{RR}$, it being at least two orders of magnitude larger than DR at any temperature of interest. Comparatively, the DR $\Delta n=1$ core excitations for 01- and 02-like are a factor 10 larger than 


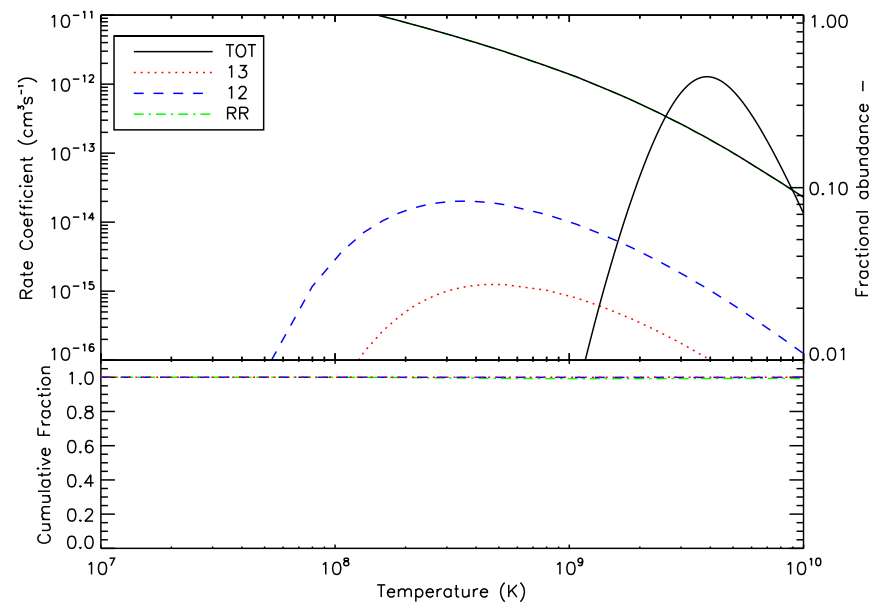

FIG. 2. 01-like DR rate coefficients for core excitations 1-2 and $1-3$, along with the RR rate coefficient, and the sum total of these. The solid black curve is the fractional abundance for 01-like as calculated using the scaled recombination data of Pütterich et al. [7] and the ionization data of Loch et al. [6]. The bottom subfigure is the cumulative sum of these different contributions (see the text for an explanation).

their corresponding $\Delta n=2$ core excitations. This is due to the (core) $n^{-3}$ scaling of the autoionization rate, rather than $\Delta n=1$ autoionization into excited states for the $\Delta n=2$. Finally, in Fig. 3 we compare the total 01- and 02-like DR rate coefficients. The 02-like is roughly a factor of 2 larger because there are two $K$-shell electrons available to promote.

\section{B. $L$-shell DR}

In Fig. 4 we have plotted the DR and RR rate coefficients for 03-like in a similar manner to Fig. 2. The RR rate coefficient drops by a factor of 2 due to the $K$-shell being closed, while dominant (for DR) contributions arise from the 2-2 and 2-3 core excitations. Nevertheless, RR still contributes $\sim 60-90 \%$ of the total recombination-rate coefficient around the temperature of peak abundance. As the $L$-shell fills, the

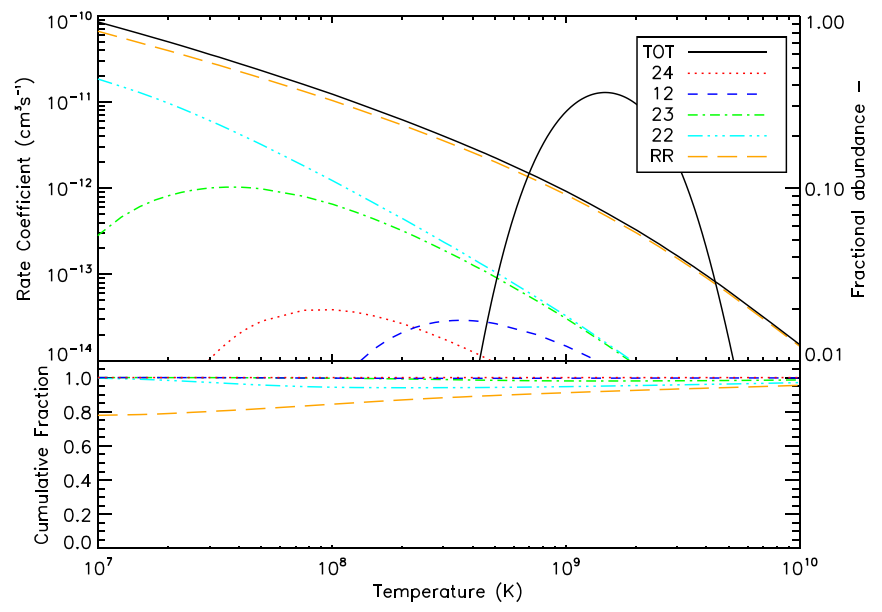

FIG. 4. As in Fig. 2, but for 03-like with core excitations 1-2, 2-2, 2-3, and 2-4.

total RR rate coefficient decreases due to decreasing $L$-shell vacancy (and charge somewhat) while the DR increases correspondingly due to the increasing number of electrons available to be promoted. The two become comparable at 10-like (see Fig. 5) when the RR can only start to fill the $M$-shell. In Figs. 6 and 7, we have plotted the DR rate coefficients for the 2-2 and 2-3 core excitations, respectively, with the former covering 03- to 09-like and the latter covering 03 - to 10-like. The 2-2 core excitation provides the largest contribution to the total DR rate coefficients when filling the $2 s$ shell. After the $2 p$ subshell is half-filled (06-like), the 2-2 DR rate coefficient decreases gradually, being overtaken by the 2-3 core excitation. The $2-4$ core excitation provides only a small contribution in 03 - and $04-$ like ( $<1 \%$ at peak abundance), and hence it was neglected from 05-like onward. In 10-like, the 2-4 core excitation was reintroduced as a consistency check now that the 2-2 is closed, however it still provides a minimal contribution of $\sim 5 \%$ around peak abundance.

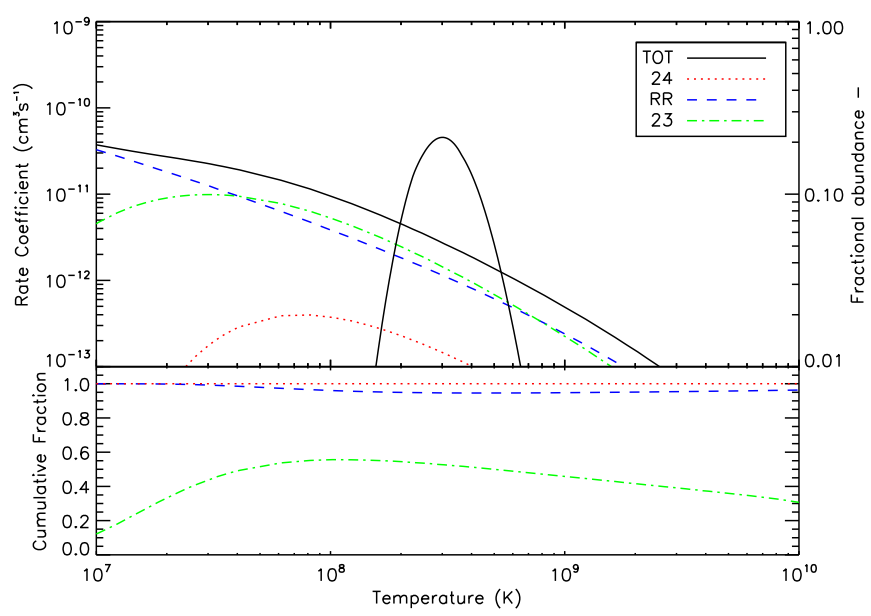

FIG. 5. As in Fig. 2, but for 10-like with core excitations 2-3 and $2-4$.
FIG. 3. DR rate coefficients for $1-2, \Delta n=1$ core excitation for

01- and 02-like.

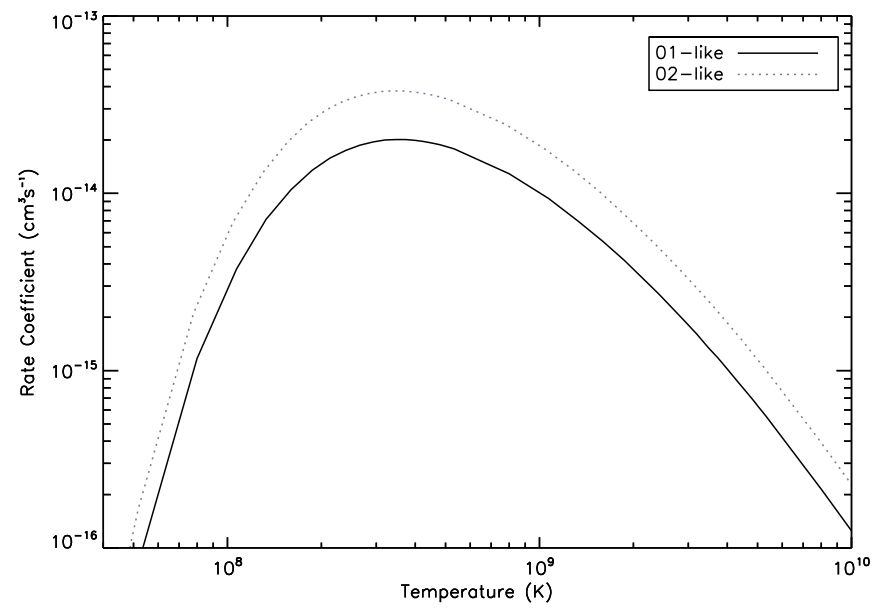




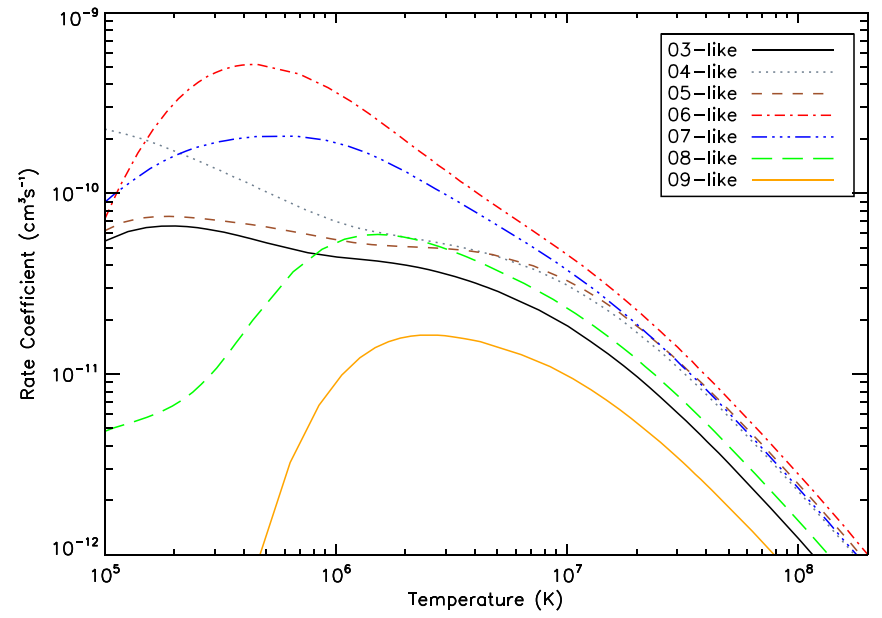

FIG. 6. DR rate coefficients for $2-2, \Delta n=0$ core excitation for 03- to 09-like.

\section{C. $M$-shell DR}

A temperature of $26 \mathrm{keV}\left(3 \times 10^{8} \mathrm{~K}\right)$ corresponds to the peak abundance of 10-like W. Higher charge states will exist, with increasingly small fractional abundance, but they may be seen spectroscopically. The $M$-shell is perhaps the deepest shell in tungsten that ITER will be able to access routinely. The $M$-shell is also the regime in which RR increasingly gives way to DR, contributing $\sim 40 \%$ of the total recombination rate coefficient in 11-like, and decreasing to $\sim 15 \%$ in 18 -like, around the temperature of peak abundance (see Figs. 8 and 9, respectively). The inner-shell 2-3 core excitation provides the largest contribution to the total DR rate coefficient in 11-like $(\sim 40 \%)$, however this is quickly overtaken by the $\Delta n=0$ and outer shell $\Delta n=1$ core excitations of 3-3 and $3-4$, respectively. Again, this can be understood in terms of a simple occupancy-vacancy argument. In addition, the 2-3 is increasingly suppressed by core re-arrangement autoionizing transitions, viz., an $M$-shell electron drops down into the $L$-shell and ejects another $M$-shell electron. This process is independent of the Rydberg- $n$, unlike the initial dielectronic

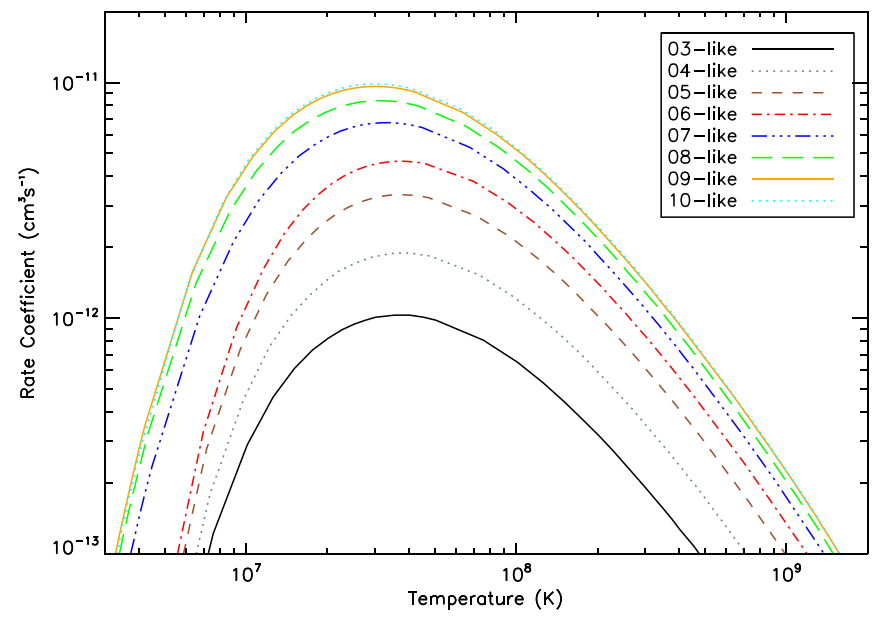

FIG. 7. DR rate coefficients for 2-3, $\Delta n=1$ core excitation for 03- to 10 -like.

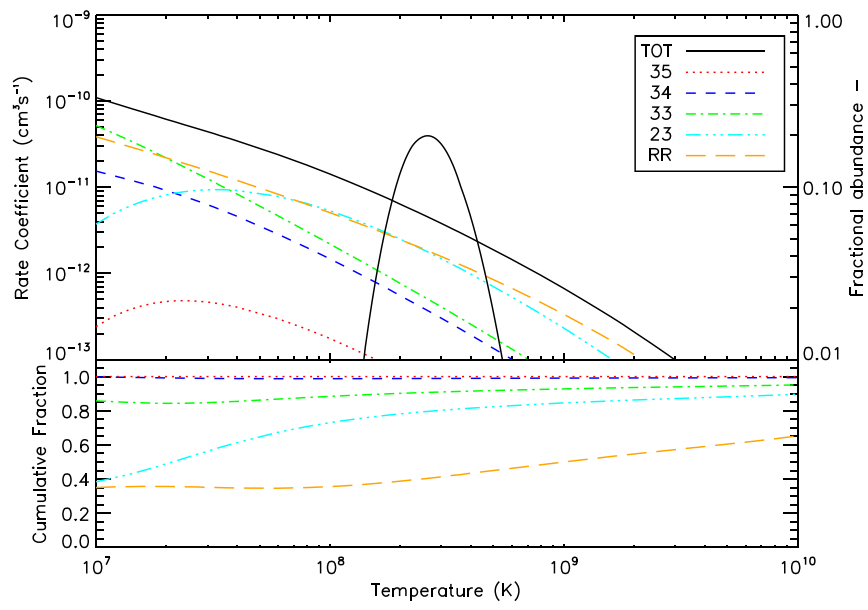

FIG. 8. As in Fig. 2, but for 11-like with core excitations 2-3, 3-3, $3-4$, and 3-5.

capture. The reduction of the 2-3 core-excitation DR with increasing $M$-shell occupation is shown in Fig. 10, where we have plotted the 2-3 DR rate coefficients for 11-like to 18-like.

The outer-shell $\Delta n=0$ (3-3) and $\Delta n=1$ (3-4) core excitations provide the largest contributions to the total recombination-rate coefficients from 12-like onward. In Fig. 11, we have plotted the $\left(3 p^{q}\right)$ 3-3 core excitations for 11 -like to 18 -like, where there is competition between $3 p$ occupancy and $3 p$ vacancy. It can be seen that the 3-3 contribution grows steadily up to 15 - and 16-like, reaching a maximum value there. The rate coefficient then begins to decrease for 17 - and 18-like as the $3 p$ shell closes, leaving only $3 d$ vacancies. In Fig. 12, we have plotted the 3-4 DR rate coefficients for 11 -like to 18 -like. The 3-4 rate coefficients increase simply with increasing $3 p$ occupancy.

The $\Delta n=2$ (3-5) core excitation again provides only a small contribution throughout 11- to 18-like. This contribution is at its smallest for 11-like (Fig. 8), contributing $\sim 1 \%$ to the total recombination-rate coefficient. As with 3-4, the 3-5 DR rate coefficient increases up to 17 - and 18-like, but it still only contributes $\sim 5 \%$ for the final ion. Despite the small

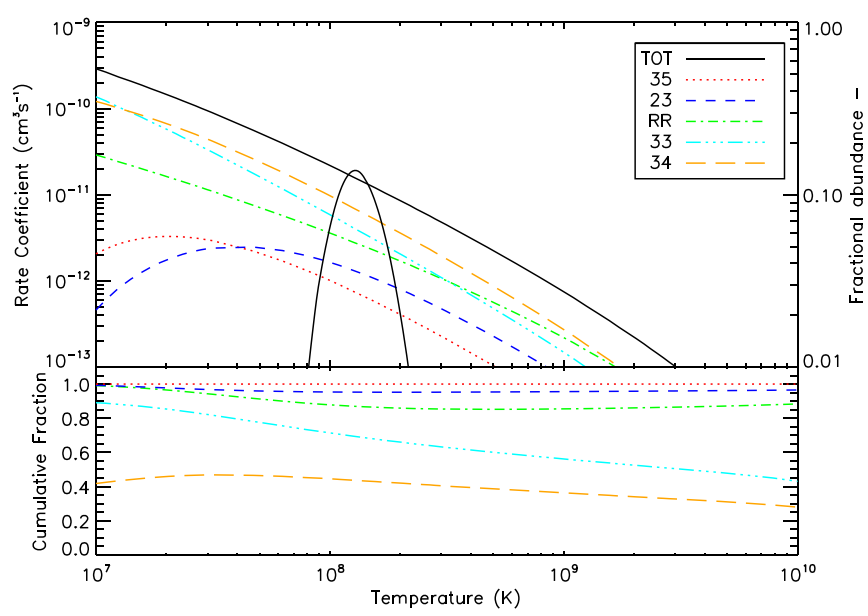

FIG. 9. As in Fig. 2, but for 18-like with core excitations 2-3, 3-3, 3-4, and 3-5. 


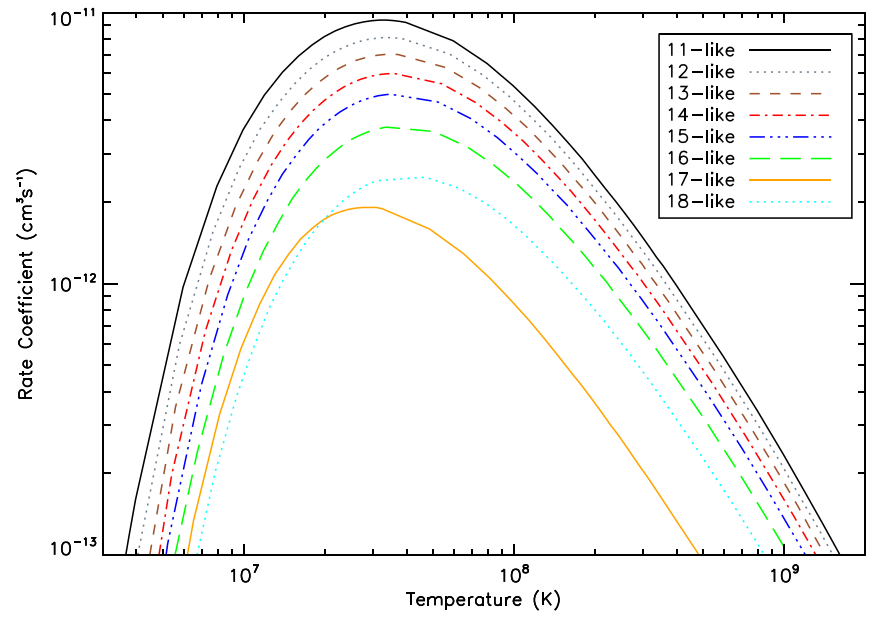

FIG. 10. DR rate coefficients for 2-3, $\Delta n=1$ core excitation for 11- to 18-like.

contribution, we opted to keep the 3-5 core excitation, as the 2-3 one decreases rapidly with the filling of the $3 p$ shell.

\section{Relativistic configuration mixing in DR}

Comparing total DR rate coefficients, although convenient, can be somewhat misleading since nonrelativistic configuration mixing and relativistic (e.g., spin-orbit) mixing are described by unitary transformations of the initial basis wave functions. For example, in Fig. 13 we show the total DR rate coefficients for the 16-like 3-4 core-excitation calculated in IC and CA. It can be seen that the agreement between IC and CA is very good, being $\sim 10 \%$ around the temperature of peak abundance. Now, if we consider a set of partial DR rate coefficients for 16-like 3-4, we can see that the agreement between IC and CA is much worse. In Fig. 14, we have plotted the partial DR rate coefficients for 16-like 3-4, capture to $n=5$. The best agreement is for recombination into the $5 f$, with IC and CA differing by $\sim 5 \%$. The worst agreement is seen for recombination into $5 p$, where the IC and CA rate coefficients differ by $\sim 33 \%$ at peak abundance. Agreement

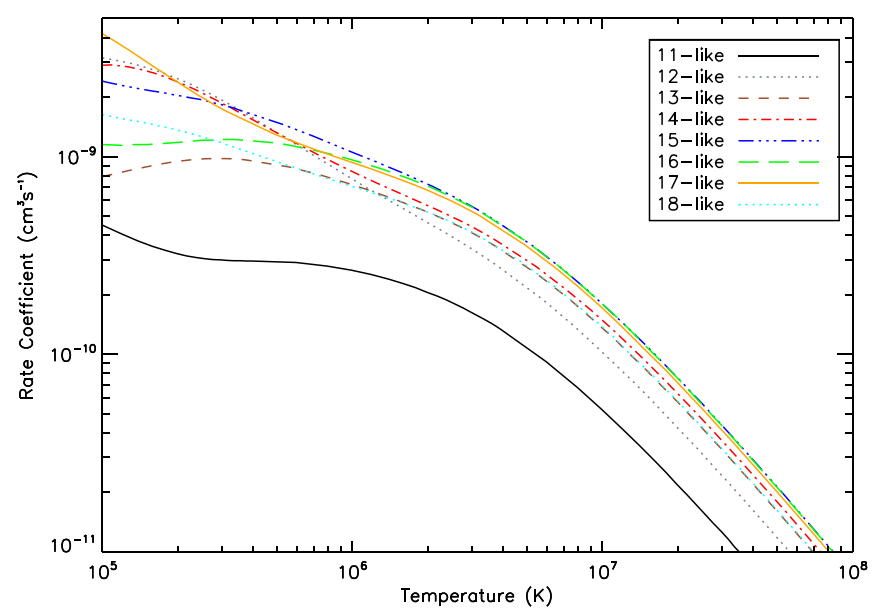

FIG. 11. DR rate coefficients for 3-3, $\Delta n=0$ core excitation for 11- to 18-like.

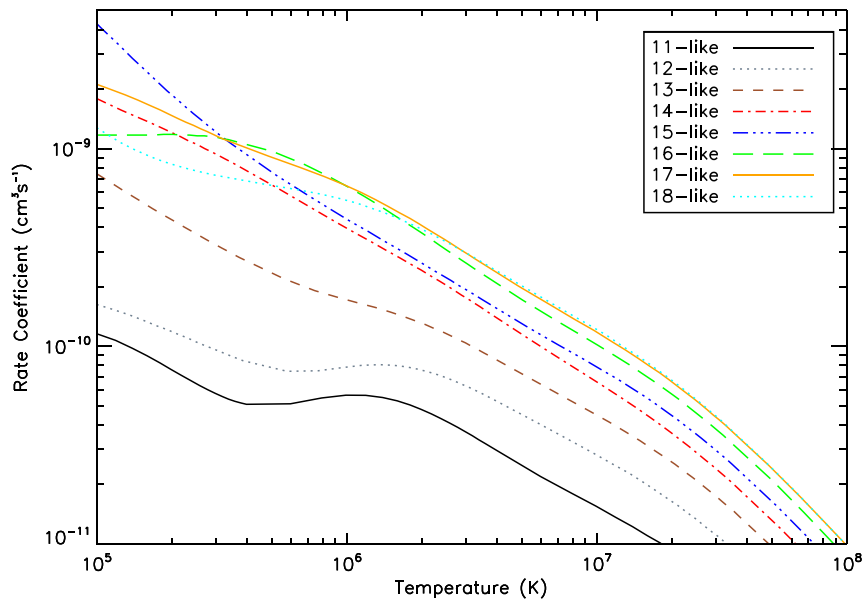

FIG. 12. DR rate coefficients for 3-4, $\Delta n=13-4$ core excitation for 11- to 18-like.

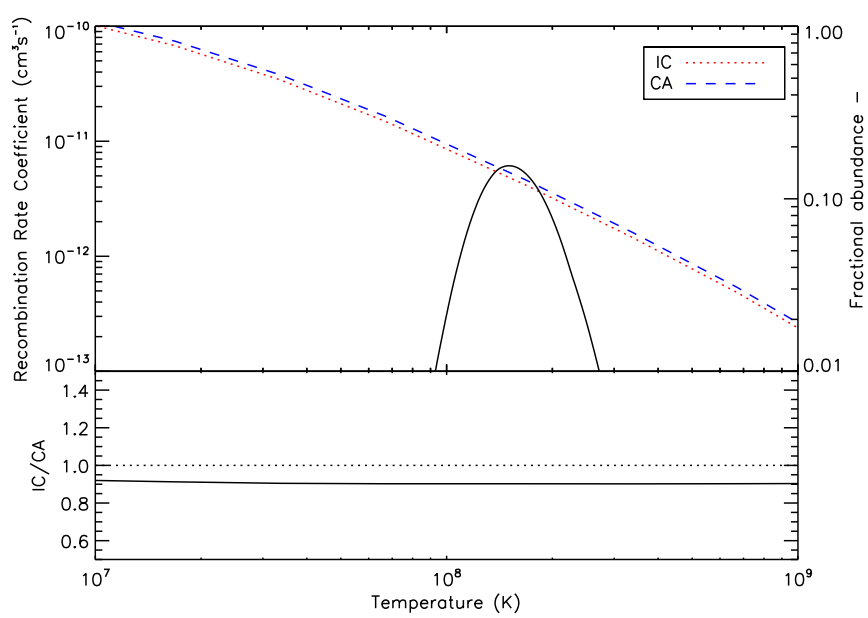

FIG. 13. Total DR rate coefficients for 16-like 3-4. The bottom plot shows the ratio of the IC coefficients to CA. The dotted line indicates a ratio of unity.

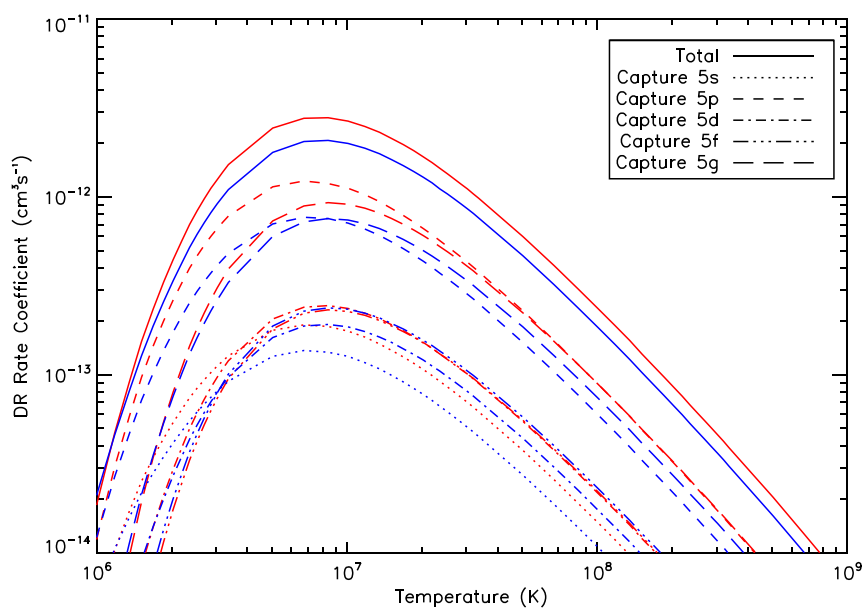

FIG. 14. Partial DR rate coefficients for 16-like 3-4 capture to $n=5$ for subshells $5 s-5 g$. The red curves correspond to the CA calculation, while the blue correspond to IC. 


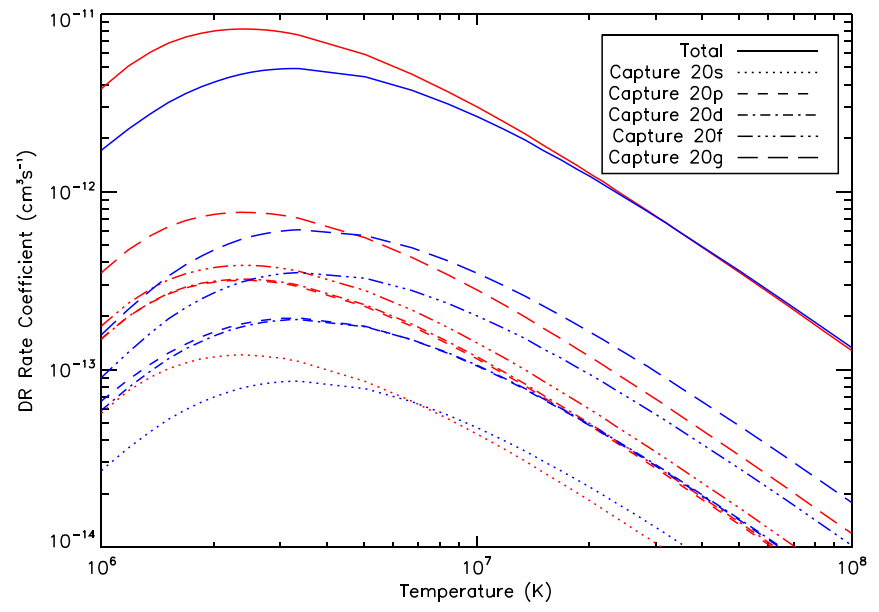

FIG. 15. Partial DR rate coefficients for 16-like 3-3 capture to $n=20$ for subshells $20 s-20 g$. The red curves correspond to the CA calculation, while the blue correspond to IC.

is no better for $5 s, 5 d$, and $5 g$, where the IC and CA rate coefficients differ by $\sim 28 \%, \sim 19 \%$, and $\sim 18 \%$, respectively.

The disagreement between partial DR rate coefficients calculated in IC and CA is even more apparent when considering the 3-3 core excitation. In Fig. 15, we have plotted the partial DR rate coefficients for 16-like 3-3, capture to $n=20$. The best agreement occurs for $20 p$ and $20 d$, where the partials differ by $<10 \%$ at peak abundance. The same cannot be said for $20 s, 20 f$, and $20 \mathrm{~g}$, where the IC and CA results differ by $\sim 30 \%, \sim 72 \%$, and $\sim 51 \%$, respectively. These differences highlight the importance of relativistic mixing for a heavy atom such as tungsten. This effect is not confined to 16-like, and it occurs for all ions considered in this work. Its subsequent propagation through collisional-radiative modeling is a topic for future study.

\section{E. Comparison with other DR work}

With the exception of closed-shell ions, not many DR rate coefficient data have been calculated for the ions $\mathrm{W}^{73+}$ to $\mathrm{W}^{56+}$. As ITER will have an operating temperature of up to $\sim 26 \mathrm{keV}\left(\sim 3 \times 10^{8} \mathrm{~K}\right)$, the reactor will be able to access to about $10-$ like $\mathrm{W}^{64+}$. In Tables III and IV, we compare our total DR rate coefficients for 10-like with those of Behar et al. [21] and Safronova et al. [20], respectively, both of whom used the HULLAC [14] code. Agreement with the results of Behar et al. is generally good, being $\sim 10 \%$ near peak abundance, while low temperatures illustrate the characteristic sensitivity of DR rate coefficients to near-threshold resonances. However, a significant difference is noted between these two sets of results and those of Safronova et al., where our DR rate coefficients are larger by $\sim 50 \%$ for temperatures $>2 \times 10^{8} \mathrm{~K}$. The origin of this difference is currently unknown. In Table $\mathrm{V}$ we compare our 18-like total DR rate coefficients with the HULLAC ones of Peleg et al. [22]. Agreement is better in this case over a wider range of temperatures, being $\lesssim 10 \%$ at peak abundance.
TABLE III. Comparison of 10-like total DR rate coefficients from this work with those of Behar et al. [21], and the \% difference between the two. ${ }^{a}$ Quantities in square brackets are powers of 10.

\begin{tabular}{lccr}
\hline \hline$T(\mathrm{~K})$ & This work & Behar et al. & \%Diff. \\
\hline $5.80[+05]$ & $4.19[-22]$ & $1.71[-21]$ & -75.5 \\
$1.16[+06]$ & $2.24[-17]$ & $5.03[-17]$ & -55.5 \\
$2.32[+06]$ & $1.11[-14]$ & $1.17[-14]$ & -5.4 \\
$5.80[+06]$ & $9.53[-13]$ & $1.34[-12]$ & -28.9 \\
$1.16[+07]$ & $4.73[-12]$ & $6.39[-12]$ & -26.0 \\
$2.32[+07]$ & $9.66[-12]$ & $1.12[-11]$ & -13.8 \\
$5.80[+07]$ & $8.71[-12]$ & $1.02[-11]$ & -14.6 \\
$1.16[+08]$ & $5.21[-12]$ & $6.06[-12]$ & -14.1 \\
$2.32[+08]$ & $2.55[-12]$ & $2.83[-12]$ & -10.0 \\
$5.80[+08]$ & $7.70[-13]$ & $8.53[-13]$ & -9.8 \\
\hline \hline
\end{tabular}

${ }^{a}$ The $\%$ difference is calculated as $\left(\alpha_{\text {DRPresent }}-\alpha_{\text {DRBehar }}\right) / \alpha_{\text {DRBehar }}$.

TABLE IV. Comparison of 10-like total DR rate coefficients from this work with those of Safronova et al. [20]. Quantities in square brackets are powers of 10 .

\begin{tabular}{|c|c|c|c|}
\hline$T(\mathrm{~K})$ & This work & Safronova et al. & $\%$ Diff. \\
\hline $6.30[+05]$ & $2.54[-21]$ & $7.67[-21]$ & -66.9 \\
\hline $8.19[+05]$ & $7.80[-19]$ & $6.66[-19]$ & 17.1 \\
\hline $1.06[+06]$ & $9.71[-18]$ & $1.97[-17]$ & -50.7 \\
\hline $1.38[+06]$ & $1.20[-16]$ & $2.61[-16]$ & -54.0 \\
\hline $1.80[+06]$ & $1.54[-15]$ & $2.01[-15]$ & -23.6 \\
\hline $2.34[+06]$ & $1.17[-14]$ & $1.14[-14]$ & 2.8 \\
\hline $3.04[+06]$ & $5.23[-14]$ & $5.40[-14]$ & -3.2 \\
\hline $3.96[+06]$ & $2.38[-13]$ & $2.12[-13]$ & 12.3 \\
\hline $5.14[+06]$ & $6.31[-13]$ & $6.47[-13]$ & -2.5 \\
\hline $6.68[+06]$ & $1.54[-12]$ & $1.53[-12]$ & 0.8 \\
\hline $8.68[+06]$ & $3.31[-12]$ & $2.86[-12]$ & 15.7 \\
\hline $1.13[+07]$ & $4.57[-12]$ & $4.42[-12]$ & 3.4 \\
\hline $1.46[+07]$ & $6.28[-12]$ & $5.89[-12]$ & 6.6 \\
\hline $1.90[+07]$ & $8.68[-12]$ & $7.00[-12]$ & 24.0 \\
\hline $2.48[+07]$ & $9.74[-12]$ & $7.60[-12]$ & 28.1 \\
\hline $3.23[+07]$ & $1.01[-11]$ & $7.68[-12]$ & 31.0 \\
\hline $4.19[+07]$ & $1.03[-11]$ & $7.29[-12]$ & 40.6 \\
\hline $5.45[+07]$ & $8.99[-12]$ & $6.54[-12]$ & 37.4 \\
\hline $7.08[+07]$ & $7.89[-12]$ & $5.58[-12]$ & 41.4 \\
\hline $9.21[+07]$ & $6.53[-12]$ & $4.56[-12]$ & 43.2 \\
\hline $1.20[+08]$ & $5.06[-12]$ & $3.59[-12]$ & 40.9 \\
\hline $1.56[+08]$ & $3.91[-12]$ & $2.73[-12]$ & 43.1 \\
\hline $2.02[+08]$ & $3.02[-12]$ & $2.03[-12]$ & 48.8 \\
\hline $2.63[+08]$ & $2.17[-12]$ & $1.48[-12]$ & 46.6 \\
\hline $3.42[+08]$ & $1.56[-12]$ & $1.06[-12]$ & 47.1 \\
\hline $4.44[+08]$ & $1.11[-12]$ & $7.47[-13]$ & 48.8 \\
\hline $5.78[+08]$ & $7.74[-13]$ & $5.22[-13]$ & 48.3 \\
\hline $7.51[+08]$ & $5.39[-13]$ & $3.62[-13]$ & 49.0 \\
\hline $9.76[+08]$ & $3.72[-13]$ & $2.50[-13]$ & 48.7 \\
\hline $1.26[+09]$ & $2.56[-13]$ & $1.71[-13]$ & 49.6 \\
\hline $1.65[+09]$ & $1.75[-13]$ & $1.17[-13]$ & 49.4 \\
\hline $2.15[+09]$ & $1.19[-13]$ & $7.96[-14]$ & 49.8 \\
\hline $2.79[+09]$ & $8.13[-14]$ & $5.41[-14]$ & 50.3 \\
\hline $3.62[+09]$ & $5.52[-14]$ & $3.67[-14]$ & 50.5 \\
\hline $4.71[+09]$ & $3.74[-14]$ & $2.49[-14]$ & 50.0 \\
\hline $6.13[+09]$ & $2.52[-14]$ & $1.69[-14]$ & 49.2 \\
\hline
\end{tabular}


TABLE V. Comparison of 18-like total DR rate coefficients from this work with those of Peleg et al. [22]. Quantities in square brackets are powers of 10 .

\begin{tabular}{llll}
\hline \hline$T(\mathrm{~K})$ & This work & Peleg et al. & $\%$ Diff. \\
\hline $1.16[+05]$ & $2.81[-09]$ & $4.50[-09]$ & -37.5 \\
$2.32[+05]$ & $2.08[-09]$ & $3.32[-09]$ & -37.2 \\
$3.48[+05]$ & $1.81[-09]$ & $2.68[-09]$ & -32.5 \\
$5.80[+05]$ & $1.52[-09]$ & $2.06[-09]$ & -26.4 \\
$1.16[+06]$ & $1.16[-09]$ & $1.45[-09]$ & -19.7 \\
$2.32[+06]$ & $8.23[-10]$ & $9.51[-10]$ & -13.4 \\
$3.48[+06]$ & $6.35[-10]$ & $7.03[-10]$ & -9.7 \\
$5.80[+06]$ & $4.24[-10]$ & $4.59[-10]$ & -7.6 \\
$1.16[+07]$ & $2.24[-10]$ & $2.45[-10]$ & -8.4 \\
$2.32[+07]$ & $1.11[-10]$ & $1.21[-10]$ & -8.1 \\
$3.48[+07]$ & $7.10[-11]$ & $7.72[-11]$ & -8.1 \\
$5.80[+07]$ & $3.84[-11]$ & $4.18[-11]$ & -8.1 \\
$8.12[+07]$ & $2.48[-11]$ & $2.72[-11]$ & -8.7 \\
$1.16[+08]$ & $1.55[-11]$ & $1.70[-11]$ & -8.9 \\
$2.32[+08]$ & $5.98[-12]$ & $6.52[-12]$ & -8.3 \\
$3.48[+08]$ & $3.36[-12]$ & $3.65[-12]$ & -7.9 \\
$5.80[+08]$ & $1.60[-12]$ & $1.74[-12]$ & -8.3 \\
\hline \hline
\end{tabular}

\section{F. $\mathbf{R R}$}

In Fig. 16, we show our total RR rate coefficients from 00like to 18-like calculated in IC. These include all multipoles up to E40/M39 and the Jütner relativistic correction. The pattern of curves seen corresponds to the filling of the $K$-shell and then the $L-/ M$-shell boundary, as noted above. As mentioned previously, Trzhaskovskaya et al. [27-30] have calculated an extensive set of partial and "total" (summed to $n=20$, $\ell=19)$ RR rate coefficients for the whole tungsten isonuclear sequence. Their calculations were fully relativistic, extending to $n=20, \ell=19$. Comparatively, our AUTOSTRUCTURE calculations extend to $n=999$ and $\ell=150$, where values up to $\ell=10$ were treated relativistically in the $\kappa$-averaged

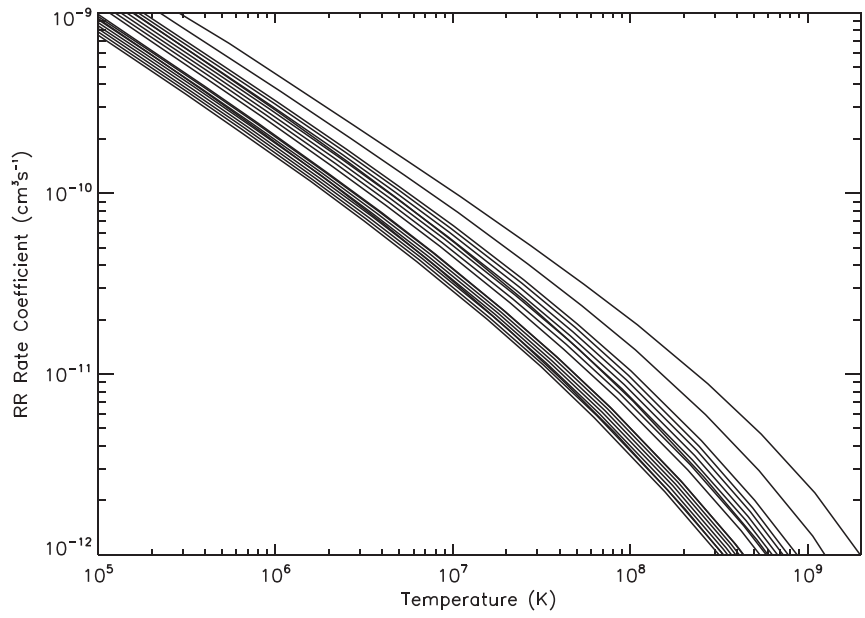

FIG. 16. Total RR rate coefficients for 00- to 18-like. The top curve is 00-like, and the curves below it are 01-like down to 18-like. All this work.

approximation. A nonrelativistic dipole top-up was then used to cover the remaining $\ell$ values, which become important at low (nonrelativistic) temperatures. In Table VI we compare the RR rate coefficients of Trzhaskovskaya et al. [27] for 00-like (fully stripped) to ours over $\log _{10} T$ (K) of 3.0 to 10.0. In this table, we have given our rate coefficients when summed up to $n=999$ and $\ell=150$, as well as the rate coefficients when summed up to $n=20$ and $\ell=19$. In the case in which we do not truncate $n$ and $\ell$, we see very large differences at low temperatures $[>100 \%$ for $\left.\log _{10} T(\mathrm{~K}) \leqslant 3.5\right]$. This difference decreases steadily until $\sim 10^{9} \mathrm{~K}$, where it then begins to increase again. When we truncate our $n$ and $\ell$ values to match those of Trzhaskovskaya et al., we find excellent agreement between the two data sets for $\log _{10} T(\mathrm{~K})<9.5(<1 \%)$. Above $\log _{10} T(\mathrm{~K})=9.5$, we note a slight drift away from the results of Trzhaskovskaya et al., reaching $\sim 10 \%$ at the highest temperature $\log _{10} T(\mathrm{~K})=10.0$.

TABLE VI. Comparison of total RR rate coefficients for 00-like between those calculated by Trzhaskovskaya et al. [27], this work, and the

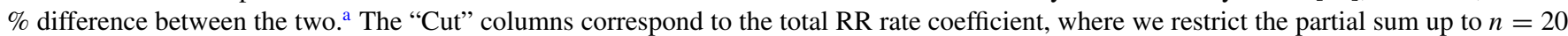
and $l=19$ so as to match that of [27]. Quantities in square brackets are powers of 10 , for example, $1.00[-1]=1.00 \times 10^{-1}$.

\begin{tabular}{|c|c|c|c|c|c|}
\hline $\log _{10} T(\mathrm{~K})$ & Trzhaskovskaya et al. & This work (No Cut) & This work (Cut) & \%Diff. (No Cut) & \%Diff. (Cut) \\
\hline 3.0 & $1.17[-08]$ & $3.00[-08]$ & $1.17[-08]$ & 156 & 0.0 \\
\hline 3.5 & $6.56[-09]$ & $1.46[-08]$ & $6.60[-09]$ & 123 & 0.6 \\
\hline 4.0 & $3.69[-09]$ & $7.28[-09]$ & $3.71[-09]$ & 97.3 & 0.5 \\
\hline 4.5 & $2.07[-09]$ & $3.64[-09]$ & $2.08[-09]$ & 75.8 & 0.5 \\
\hline 5.0 & $1.16[-09]$ & $1.83[-09]$ & $1.17[-09]$ & 57.8 & 0.9 \\
\hline 5.5 & $6.45[-10]$ & $9.09[-10]$ & $6.48[-10]$ & 40.9 & 0.5 \\
\hline 6.0 & $3.51[-10]$ & $4.47[-10]$ & $3.53[-10]$ & 27.4 & 0.6 \\
\hline 6.5 & $1.85[-10]$ & $2.16[-10]$ & $1.86[-10]$ & 16.8 & 0.5 \\
\hline 7.0 & $9.30[-11]$ & $1.02[-10]$ & $9.35[-11]$ & 9.7 & 0.5 \\
\hline 7.5 & $4.41[-11]$ & $4.64[-11]$ & $4.43[-11]$ & 5.2 & 0.5 \\
\hline 8.0 & $1.95[-11]$ & $2.00[-11]$ & $1.95[-11]$ & 2.6 & 0.0 \\
\hline 8.5 & $7.71[-12]$ & $7.80[-12]$ & $7.69[-12]$ & 1.2 & -0.3 \\
\hline 9.0 & $2.46[-12]$ & $2.46[-12]$ & $2.44[-12]$ & 0.0 & -0.8 \\
\hline 9.5 & $5.42[-13]$ & $5.26[-13]$ & $5.24[-13]$ & -3.0 & -3.3 \\
\hline 10.0 & $7.86[-14]$ & $7.12[-14]$ & $7.10[-14]$ & -9.4 & -9.7 \\
\hline
\end{tabular}

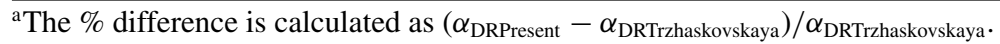




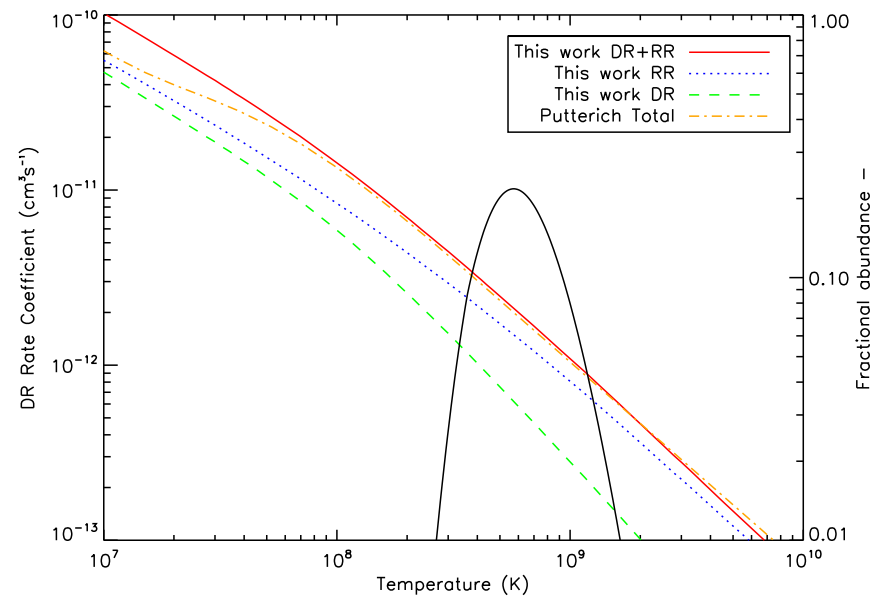

FIG. 17. Comparison of the present separate DR and RR rate coefficients, together with their sum total, with the total rate coefficients of Pütterich et al. [7] for 06-like.

This is likely due to the use of $\kappa$-average wave functions by AUTOSTRUCTURE, assuming $<1 \%$ accuracy in the results of Trzhaskovskaya et al. still. The $\kappa$-average approximation begins to break down at high temperatures, or rather at the corresponding high electron energies that contribute at such $T$. The underlying photoionization cross sections are falling-off rapidly in magnitude, and such small quantities become increasingly sensitive to the $\kappa$-average approximation. Such a difference at these temperatures should be of no importance to modeling. However, it is useful to have a complete set of consistent partial RR data to complement the DR data for collisional-radiative modeling with ADAS. As already noted, $\mathrm{RR}$ is most important for the highest few ionization stages. By 10-like, the total DR rate coefficient is comparable to RR at the temperature of peak abundance. By 18-like, the RR rate coefficient contributes only $\approx 10 \%$ to the total rate coefficient at peak abundance.

\section{G. Comparison with Pütterich et al. and Foster DR+RR}

The Pütterich et al. [7] DR data are ADPAK [9,10], which uses an average-atom method, and they were further scaled for $\mathrm{W}^{22+}-\mathrm{W}^{55+}$. The Foster [8] DR data were calculated using the Burgess General Formula [11]. Both use the same ADAS RR data, which are scaled hydrogenically. We now compare our $\mathrm{DR}+\mathrm{RR}$ results with those Pütterich et al. and Foster. To do this, we omit the Jüttner relativistic correction from our recombination-rate coefficients, as they did. Upon comparing our recombination-rate coefficients with those of Pütterich et al., we find that there are multiple ions for which there is good agreement. For example, in Fig. 17 we have plotted the 06-like recombination-rate coefficients for Pütterich et al., and our DR and RR rate coefficients and their sum. We find that our rate coefficients are in agreement with those of Pütterich et al. to $<10 \%$ at peak abundance. Some ions are in poor agreement. In Fig. 18, we compare our recombination-rate coefficients with those of Pütterich et al. for 10-like. The agreement is very poor at peak abundance with a difference of $>40 \%$. For the Foster data, good agreement is again seen in multiple ions. In Fig. 19, we plot our DR, RR, and

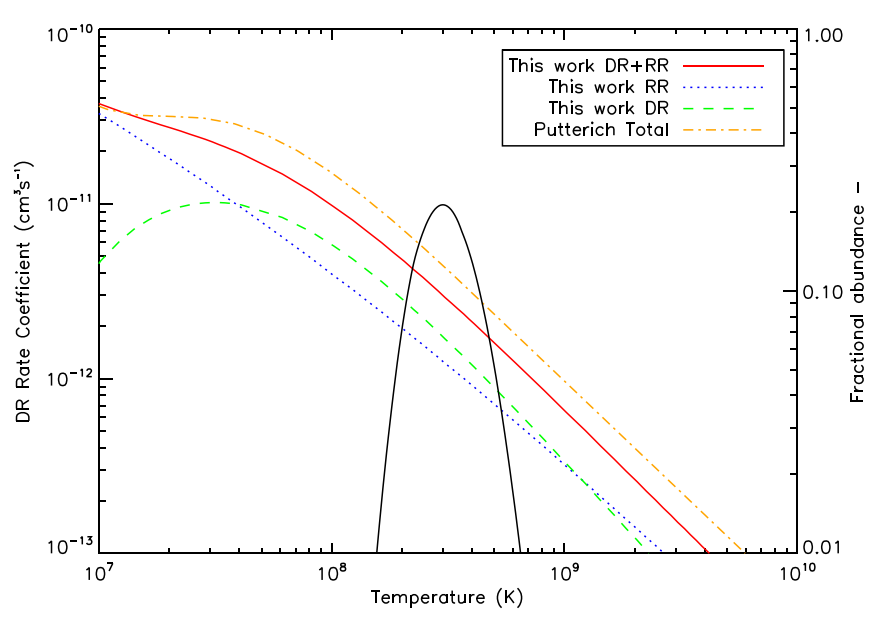

FIG. 18. As in Fig. 17, but for 10-like.

total recombination-rate coefficients along with Foster's total $(\mathrm{DR}+\mathrm{RR})$ rate coefficients for 06-like. The difference between ours and Foster's rate coefficient is even smaller than that found with Pütterich et al., being $<1 \%$ at peak abundance. The largest disagreement between ours and Foster's data occurs for 16-like. We have plotted ours and Foster's recombination-rate coefficients for 16-like in Fig. 20. Poor agreement can be seen across a wide temperature range. At peak abundance, our and Foster's recombination-rate coefficients differ by $>40 \%$.

The agreement between our present total DR plus RR rate coefficients and those of Foster [8] is similar to the agreement between ours and those of Pütterich et al. [7] for 01-like to 11-like, with the differences being $<30 \%$ near peak abundance. For 12-like and beyond, the recombination data of Pütterich et al. are in better agreement with ours, while Foster's data are consistently smaller than ours. As previously noted, DR becomes increasingly important as we move from the $L$-shell to the $M$-shell. Thus, crude or simple methods such as average atom and the Burgess General Formula can give good descriptions of DR, but also very poor ones. Also, they are not readily adaptable to delivering the partial final-state-resolved

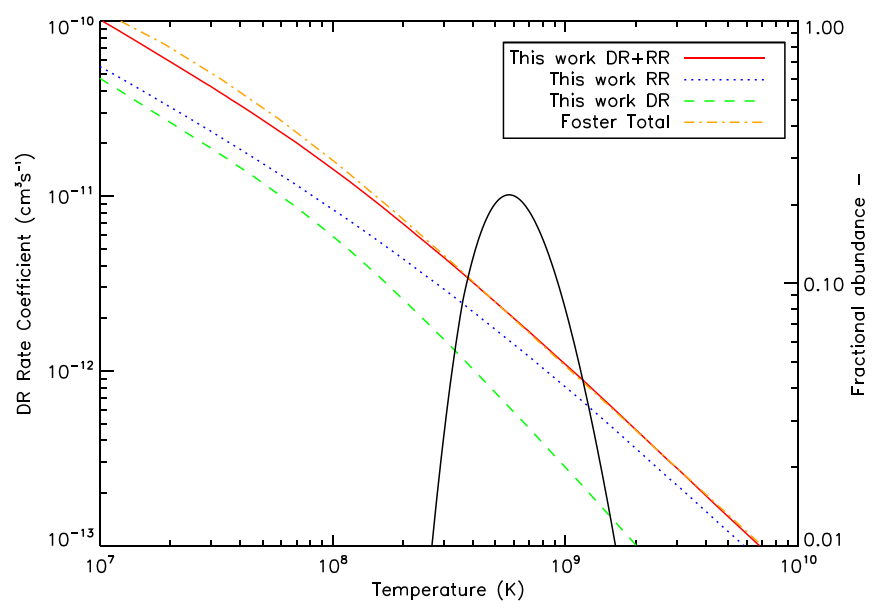

FIG. 19. Comparison of the present separate DR and RR rate coefficients, together with their sum total, with the total rate coefficients of Foster [8] for 06-like. 


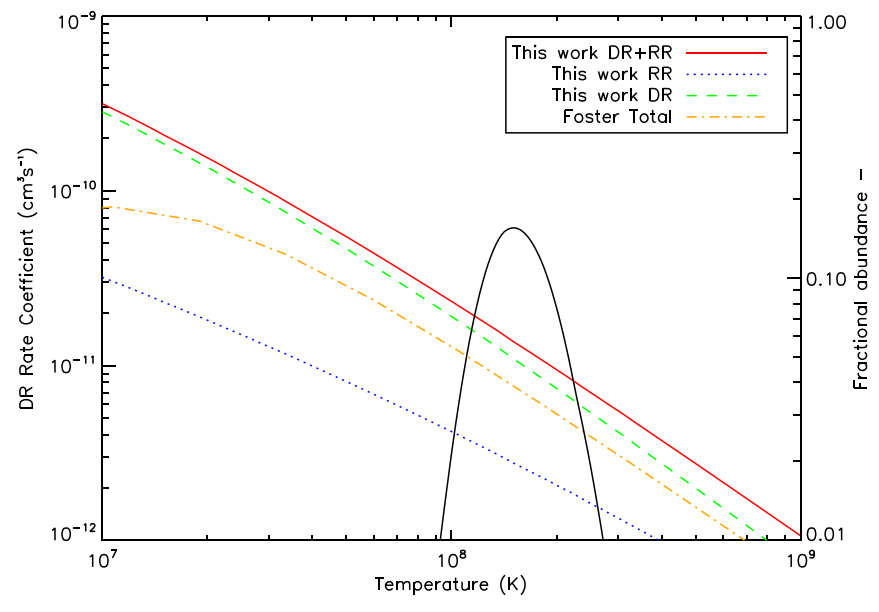

FIG. 20. Same as Fig. 19, but for 16-like.

data required for collisional-radiative modeling, although the Burgess General Program underlying his General Formula can do so.

\section{H. Ionization balance}

To compare the effect of our new recombination data, on the zero-density ionization balance, with those of Pütterich et al. [7], we replaced their recombination data with our new DR+RR data for 00-like to 18-like tungsten. In Fig. 21, we compare the ionization balance obtained with this new dataset with the original one of Pütterich et al. A large discrepancy is immediately apparent, namely that our peak abundance fractions have shifted relative to those of Pütterich et al. This has a simple explanation, in that our data have the Jütner relativistic correction applied. By excluding this correction, our ionization fraction moves into better agreement with the Pütterich et al. fraction, as seen in Fig. 22.

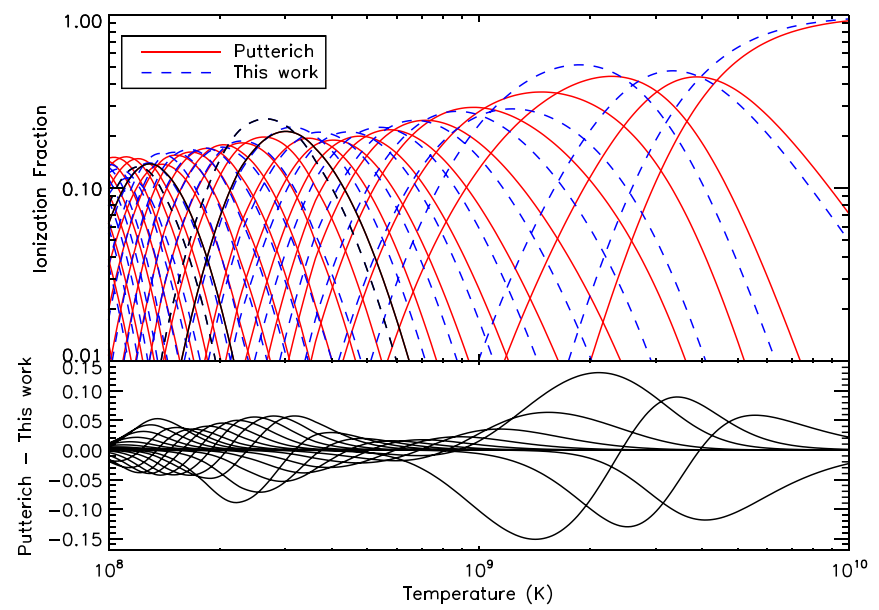

FIG. 21. Zero-density fractional abundances of tungsten ionization stages, calculated using the recombination data of Pütterich et al. [7] (red, solid curves) and the present recombination data (blue, dashed curves). The black curves, from right to left, indicate 10-like and 18-like. Both use the ionization rate coefficients from Loch et al. [6].

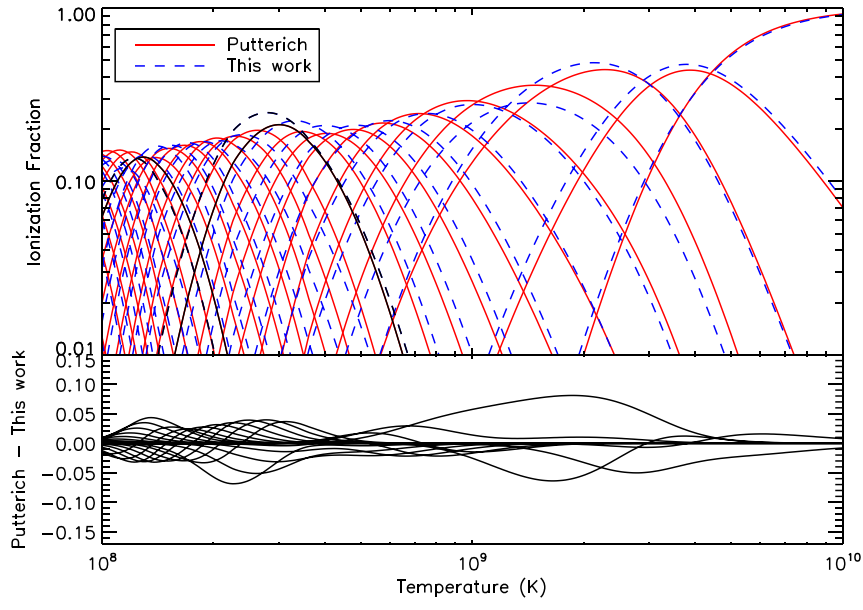

FIG. 22. As in Fig. 21, but with the Jüttner correction removed from our data.

Electric and magnetic multipole radiation contributions to the RR rate coefficients become important at high temperatures [42]. In Fig. 23, we have plotted the ionization balance using RR rate coefficients where only electric dipole radiation is included, and using $\mathrm{RR}$ rate coefficients where electric and magnetic multipoles up to E40 and M39 have been included. The inclusion of higher multipoles increases the peak abundance temperature of the highest-charge ions as expected, however the peak abundance temperature only changes by $\sim 4 \%$ for $01-$ and $02-$ like. This shift decreases rapidly to zero toward 18-like as DR becomes dominant over RR.

\section{CONCLUSION}

Large uncertainties exist in the tungsten ionization balance over a wide range of temperatures (charge-states) found in magnetic fusion plasmas. This ranges from the cool edge plasma right through to the hot core plasma. The cause is

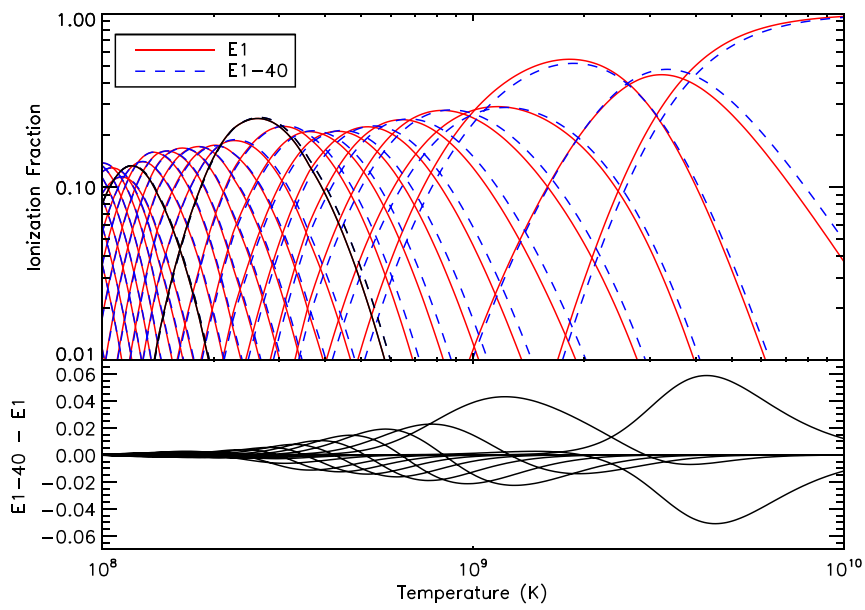

FIG. 23. Zero-density fractional abundances of tungsten ionization stages, calculated using RR rate coefficients with dipole only (red, solid curves) and E1-40/M39 multipoles included (blue, dashed curves). All this work. 
the simplified treatment of DR, using either the average-atom or Burgess General Formula approaches. We have embarked on a program of work to address this deficiency. In this paper, we have reported on the calculation of CA \& IC DR and RR rate coefficients for 00 -like to 18 -like tungsten $\left(\mathrm{W}^{74+}\right.$ to $\mathrm{W}^{56+}$ ions) using AUTOSTRUCTURE. In particular, we retain the partial final-state-resolved coefficients in a suitable form (adf09 and adf48 files), which are necessary for the collisional-radiative modeling of tungsten ions at the densities found in magnetic fusion plasmas.

We have compared our total DR rate coefficients to the results of calculations provided by Behar et al. [21] and Safronova et al. [20] for 10-like, and Peleg et al. [22] for 18 -like tungsten. Good agreement is found between our rate coefficients and those of [21] and [22] for 10-like and 18-like, differing by $\sim 10 \%$ at the peak abundance temperature. Poor agreement was found when comparing with the 10-like results of [20], with differences of $\sim 50 \%$.

RR dominates the recombination of the highest charge states ( $K$-shell ions) but DR becomes increasingly important as the $L$-shell fills and by 10 -like it is (just) larger around the temperatures of peak abundance. For more lowly ionized tungsten, beyond 10-like, the importance of RR rapidly diminishes.

We have calculated a new zero-density ionization balance for tungsten by replacing the Pütterich et al. [7] recombination with our new DR+RR data for 00-like to 18-like. Large differences result, both in the peak abundance temperatures and the ionization fractions, due largely to our inclusion of the Jüttner relativistic correction to the Maxwell-Boltzmann electron distribution. A further, smaller, difference arises from our inclusion of high electric and magnetic multipole radiation, which causes a slight shift in the peak abundance temperatures of higher ionization stages (in particular, $K$-shell ions).

This paper has presented the first step in a larger programme of work within The Tungsten Project. The next paper will cover DR/RR calculations for 19-like to 36-like tungsten, with the possibility of modeling a density-dependent ionization balance. Our ultimate goal within The Tungsten Project is to calculate partial and total DR/RR rate coefficients for the entire isoelectronic sequence of tungsten. This will replace the less reliable data used at present, which are mostly based on average-atom and the Burgess General Formula (for DR), and which give rise to large uncertainties in the tungsten ionization balance.

\section{ACKNOWLEDGMENTS}

This work was supported by the Engineering and Physical Sciences Research Council (EPSRC), Grant No. EP/1021803 to the University of Strathclyde. One of us (S.P.P.) would like to thank Stuart Henderson, Stuart Loch, and Connor Ballance for useful discussions.
[1] http://www.iter.org.

[2] ITER Physics Basis Editors, ITER Physics Expert Group Chairs, and Co-Chairs, and ITER Joint Central Team and Physics Integration Unit, Nucl. Fusion 39, 2137 (1999).

[3] A. El-Kharbachi, J. Chêne, S. Garcia-Argote, L. Marchetti, F. Martin, F. Miserque, D. Vrel, M. Redolfi, V. Malard, C. Grisolia, and B. Rousseau, Int. J. Hydrogen Energy 39, 10525 (2014).

[4] N. Fedorczak, P. Monier-Garbet, T. Pütterich, S. Brezinsek, P. Devynck, R. Dumont, M. Goniche, E. Joffrin, E. Lerche, B. Lipschultz, E. de la Luna, G. Maddison, C. Maggi, G. Matthews, I. Nunes, F. Rimini, E. R. Solano, P. Tamain, M. Tsalas, and P. de Vries, J. Nucl. Mater. 463, 85 (2015).

[5] A. P. Zakharov, V. M. Sharapov, and E. I. Evko, Soviet materials science: A translation of Fiziko-khimicheskaya mekhanika materialov/Acad. Sci. Ukrainian SSR 9, 149 (1975).

[6] S. D. Loch, J. A. Ludlow, M. S. Pindzola, A. D. Whiteford, and D. C. Griffin, Phys. Rev. A 72, 052716 (2005).

[7] T. Pütterich, R. Neu, R. Dux, A. D. Whiteford, M. G. O’Mullane, and the ASDEX Upgrade Team, Plasma Phys. Controlled Fusion 50, 085016 (2008).

[8] A. R. Foster, On the behaviour and radiating properties of heavy elements in fusion plasmas, Ph.D. thesis, University of Strathclyde, http://www.adas.ac.uk/theses/foster_thesis.pdf (2008).

[9] D. E. Post, R. V. Jensen, C. B. Tarter, W. H. Grasberger, and W. A. Lokke, At. Data Nucl. Data Tables 20, 397 (1977).

[10] D. Post, J. Abdallah, R. E. H. Clark, and N. Putvinskaya, Phys. Plasmas 2, 2328 (1995).
[11] A. Burgess, Astrophys. J. 141, 1588 (1965).

[12] N. R. Badnell, C. P. Ballance, D. C. Griffin, and M. O'Mullane, Phys. Rev. A 85, 052716 (2012).

[13] K. Spruck, N. R. Badnell, C. Krantz, O. Novotný, A. Becker, D. Bernhardt, M. Grieser, M. Hahn, R. Repnow, D. W. Savin, A. Wolf, A. Müller, and S. Schippers, Phys. Rev. A 90, 032715 (2014).

[14] A. Bar-Shalom, M. Klapisch, and J. Oreg, J. Quant. Spectrosc. Radiat. Transf. 71, 169 (2001).

[15] R. D. Cowan, The Theory of Atomic Structure and Spectra, Los Alamos Series in Basic and Applied Sciences (University of California Press, Berkeley, 1981).

[16] U. I. Safronova, A. S. Safronova, and P. Beiersdorfer, J. Phys. B 45, 085001 (2012).

[17] U. I. Safronova, A. S. Safronova, P. Beiersdorfer, and W. R. Johnson, J. Phys. B 44, 035005 (2011).

[18] U. I. Safronova and A. S. Safronova, Phys. Rev. A 85, 032507 (2012).

[19] U. I. Safronova, A. S. Safronova, and P. Beiersdorfer, J. Phys. B 42, 165010 (2009).

[20] U. I. Safronova, A. S. Safronova, and P. Beiersdorfer, At. Data Nucl. Data Tables 95, 751 (2009).

[21] E. Behar, P. Mandelbaum, and J. L. Schwob, Phys. Rev. A 59, 2787 (1999).

[22] A. Peleg, E. Behar, P. Mandelbaum, and J. L. Schwob, Phys. Rev. A 57, 3493 (1998).

[23] M. F. Gu, Astrophys. J. 590, 1131 (2003).

[24] F.-C. Meng, L. Zhou, M. Huang, C.-Y. Chen, Y.-S. Wang, and Y.-M. Zou, J. Phys. B 42, 105203 (2009). 
[25] B. W. Li, G. O’Sullivan, Y. B. Fu, and C. Z. Dong, Phys. Rev. A 85, 052706 (2012).

[26] Z. Wu, Y. Fu, X. Ma, M. Li, L. Xie, J. Jiang, and C. Dong, Atoms 3, 474 (2015).

[27] M. B. Trzhaskovskaya, V. K. Nikulin, and R. E. H. Clark, At. Data Nucl. Data Tables 96, 1 (2010).

[28] M. B. Trzhaskovskaya and V. K. Nikulin, At. Data Nuclear Data Tables 99, 249 (2013).

[29] M. B. Trzhaskovskaya and V. K. Nikulin, At. Data Nucl. Data Tables 100, 986 (2014).

[30] M. B. Trzhaskovskaya and V. K. Nikulin, At. Data Nucl. Data Tables 100, 1156 (2014).

[31] http://www.adas.ac.uk.

[32] N. R. Badnell, J. Phys. B 19, 3827 (1986).

[33] N. R. Badnell, J. Phys. B 30, 1 (1997).
[34] N. R. Badnell, Comput. Phys. Commun. 182, 1528 (2011).

[35] M. S. Pindzola, N. R. Badnell, and D. C. Griffin, Phys. Rev. A 46, 5725 (1992).

[36] N. R. Badnell, Astrophys. J. Suppl. 167, 334 (2006).

[37] I. P. Grant, J. Phys. B 7, 1458 (1974).

[38] J. L. Synge, The Relativistic Gas, Series in Physics (NorthHolland, Amsterdam, 1957).

[39] N. R. Badnell, M. G. O'Mullane, H. P. Summers, Z. Altun, M. A. Bautista, J. Colgan, T. W. Gorczyca, D. M. Mitnik, M. S. Pindzola, and O. Zatsarinny, Astron. Astrophys. 406, 1151 (2003).

[40] http://open.adas.ac.uk/.

[41] W. Eissner and H. Nussbaumer, J. Phys. B 2, 1028 (1969).

[42] R. H. Pratt, A. Ron, and H. K. Tseng, Rev. Mod. Phys. 45, 273 (1973). 